\title{
Mathematical analysis of a Markovian multi-server feedback queue with a variant of multiple vacations, balking and reneging
}

\author{
Amina Angelika Bouchentouf ${ }^{1}$, Latifa Medjahri ${ }^{2}$, \\ Mohamed Boualem ${ }^{3}$, Amit Kumar ${ }^{4}$ \\ ${ }^{1}$ Djillali Liabes University of Sidi Bel Abbes, 22000, Algeria \\ ${ }^{2}$ University of Tlemcen, B.P. 119, 13000, Algeria \\ ${ }^{3}$ University of Bejaia, 06000, Algeria \\ ${ }^{4}$ Chandigarh University, Mohali (Punjab), Pin No-140413, India
}

(received: November 26, 2021; revised: December 20, 2021; accepted: February 18, 2022)

\begin{abstract}
In this paper, we analyze a multi-server queue with customers' impatience and Bernoulli feedback under a variant of multiple vacations. On arrival, a customer decides whether to join or balk the system, based on the observation of the system size as well as the status of the servers. It is supposed that customer impatience can arise both during busy and vacation period because of the long wait already experienced in the system. The latter can be retained via certain mechanism used by the system. The feedback occurs as returning a part of serviced customers to get a new service. The queue under consideration can be used to model the processes of information transmission in telecommunication networks. We develop the Chapman-Kolmogorov equations for the steady-state probabilities and solve the differential equations by using the probability generating function method. In addition, we obtain explicit expressions of some important system characteristics. Different queueing indices are derived such as the probabilities when the servers are in different states, the mean number of customers served per unit of time, and the average rates of balking and reneging.
\end{abstract}

Key words and phrases: Markovian multi-server queue, probability generating function, impatient phenomena, server vacations, Bernoulli feedback

\section{Introduction}

Queueing models with server vacation have been efficiently studied by many researchers in the last decades and successfully applied in various practical problems such as telecommunication system design and control, manufacturing industries, and other related systems. There are two basic vacation queueing models namely, multiple vacation, and single vacation. In multiple vacation

(C) Bouchentouf A. A., Medjahri L., Boualem M., Kumar A., 2022

This work is licensed under a Creative Commons Attribution 4.0 International License http://creativecommons.org/licenses/by/4.0/ 
queueing models, the server continues to take successive vacations until it finds at least one customer waiting in a queue at a vacation completion epoch [1], [2]. Nevertheless, in single vacation queueing models, the server precisely takes one vacation between two consecutive busy periods. These two types of vacation models were first introduced by Levy and Yechiali [3]. Eminent literature on the subject is found in [4]-[8] and others.

Over the past few years, queueing models with Bernoulli feedback have increasingly attracted the attention of many researchers [9]-[14]. Taking into account the feedback effect makes it possible to bring the considered models closer to a real situation, where the claims once serviced may require repeat service for different reasons. For example, in communication networks erroneously transmitted, a data is retransmitted.

In recent years, a growing body of literature has emerged on the analysis of queueing systems with impatient customers [15]. This is due to their potential applications in many related areas, see for instance [16], [17]. Balking is one form of impatience, which is the reluctance of a customer to join a queue upon arrival [18], [19]. The other forms are reneging, the reluctance to remain in line after joining and waiting, and jockeying between lines when each of a number of parallel lines has its own queue [20], [21]. When the impatience becomes sufficiently strong, the manager of the firm concerned has to take some measures to diminish the congestion to levels that customers can tolerate.

In most queueing situations, customers seem to get discouraged from receiving service when the server is absent and tend to leave the system without receiving service. This phenomenon is very precisely observed when the server is on vacation. This results in a potential loss of customers and customer goodwill for a service provider. For a comprehensive overview of the subject, authors may refer to [22]-[29]. Most of the literature mentioned here studies reneging during the vacation state of the server. However, in many real-life situations, the abandonment may occur even when the system is in the busy state. For instance, incoming customers can not have any information about the state of the server, or when they are not satisfied with the service time (in particular, when they find that the server takes too much time to serve the customers). This paper contributes in this sense. In fact, only a few research papers have been done treating this case [9]-[12], [30].

In this paper, we provide the analysis of a multi-server feedback queue with a variant multiple vacation policy, balking and server's states-dependent reneging. When all the customers present in the system have been served, the servers immediately leave for a vacation. If they return from a vacation to find an empty queue, they leave for another vacation; otherwise, the servers, synchronously, return to serve the queue. These latter are permitted to take a finite number, say $K$, of sequential vacations. It is assumed that an arriving customer who finds the system (all the servers) on vacation period (respectively, on busy period) activates an impatience timer $T_{\text {Vac }}$ (respectively, $\left.T_{\text {Busy }}\right)$. If the customer's service has not been completed before the customer's impatience timer expires, the customer abandons the queue. The latter can be convinced to stay in the system (retained) using certain strategy. In addition, if the customer is unhappy with the service, he can rejoin the end of the queue for another one with some probability. That's what we call a feedback customer. To the best of the researchers' knowledge, the 
model under consideration has so far not treated in the literature of queues. Moreover, our model can be considered as a generalized version of existing queueing model given by Yue et al. [27] and [20] equipped with many features and associated with many practical situations.

The rest of the paper is arranged as follows. In Section 2, we introduce the mathematical description of the model and we give a practical application. In Section 4, we develop the differential equations for the probability generating functions of the steady-state probabilities. In Section 5, we give the solution of the differential equations. In Section 6, we give the probabilities when the servers are in different states. Some essential system performance measures of this model are obtained in Section 7. Finally, we conclude the paper in Section 8.

\section{The mathematical description of the model}

We consider a multi-server feedback queueing system with $K$-variant vacation, balking and server's states-dependent reneging. The following assumptions and notations are taken into account to structure the proposed queueing system:

1. The suggested queueing system consists of $c$ servers. Customers arrive into the system according to a Poisson process with rate $\lambda>0$, they are served according to First-Come-First-Served (FCFS) discipline. The service times are assumed to be exponentially distributed with rate $\mu$.

2. A multiple synchronous vacation policy is considered; once all the customers present in the system are served, the servers, all together, leave for a vacation. At the end of the vacation period, if the queue is still empty, they immediately leave for another vacation; otherwise, they return to serve the queue. The servers are allowed to take all together $K$ vacations sequentially. When the $K$ consecutive vacations are complete, the servers switch to a busy period and, depending on the arrival of new customers, they stay idle or busy. The vacation period is assumed to be exponentially distributed with rate $\phi$.

3. Whenever a customer arrives at the system and finds the servers on vacation period (resp. busy period), it activates an impatience timer $T_{\text {Vac }}$ (resp. $T_{\text {Busy }}$ ), which is exponentially distributed with parameter $\xi_{0}$ (resp. $\xi_{1}$ ). If the customer's service has not been completed before the customer's timer expires, this later may leave the system. We suppose that the customers timers are independent and identically distributed random variables and independent of the number of waiting customers.

4. It is supposed that a system employs a certain mechanism in order to keep impatient customers in the system, that is, with some probability $\alpha^{\prime}$, a customer may be retained in the system, and with a complementary probability $\alpha$ it may decide to leave to never return.

5. If, after completion of service, a customer is not happy with the quality of the service, he can return to the system with some probability $\beta^{\prime}$ for another service, or decide to leave the system with probability $\beta=1-\beta^{\prime}$.

6. A customer who on arrival finds at least one customer (resp. $c$ customers) in the system, when the servers are on vacation period (resp. busy 
period) either decides to enter the queue with probability $\theta$ or balk with probability $\bar{\theta}=1-\theta$.

All random variables presented above are mutually independent of each other.

\section{Practical application of the model}

The operation mode of a call center with vacation and impatience provides an initial motivation for our study; a central office is used for receiving or transmitting a large volume of enquiries. A private branch exchange (PBX) is a private telephone network used within a company or organizations that offers various features such as transfer calls, voicemail, call recording, interactive voice menus (IVR), and call queues. It helps in making an organization's communication simpler and more robust.

The incoming calls are routed to an available customer support manager drawn from the group of agents. Assume that the service facility consists in a group of $c$ channels (servers) available to meet the demands of the requests. If an arriving call finds some servers free it immediately occupies the channel and leaves the system after service. However, the behavior of a call may vary depending on the waiting expectations provided by the call center and the personal preferences of each specific customer. Therefore, each call may decide either to balk or to wait for a while.

The servers commute between busy and vacation periods in groups. When there is no demands to be handled, the latter, all together, go synchronously on vacation and come back as one station to the busy period, once the idle period ends. If there are some waiting calls at the end of the vacation period, they will be immediately served. Alternatively, they quit for another vacation period.

The calls have no information on the queue length nor the state of the servers, then, an increase in the mean waiting time of a customer in the system can anticipate an increase in the average rate of reneging. Thus, to avoid losing potential customers, the system should employ some strategies by choosing the system parameter to further encourage customers to stay in the system. In the case that the service is not successful, the customer can repeat its request again and again until the service succeeds.

\section{Governing equations}

At an arbitrary time, the system state is defined by a continuous time Markov chain $\{(L(t) ; J(t)) ; t \geqslant 0\}$ on the state space $\Omega=\{(n ; j)$ : $n \geqslant 0 ; j=\overline{0, K}\}$, where $L(t)$ is the number of customers in the system and $J(t)$ is the state of the servers, i.e.,

$$
J(t)= \begin{cases}j, & \text { if the servers are taking the }(j+1)^{\text {th }} \text { vacation at time } t \\ & j=\overline{0, K-1}, \\ K, & \text { if the servers are idle or busy at time } t\end{cases}
$$


Let $P_{n, j}=\lim _{t \rightarrow \infty} \mathbb{P}(L(t)=n ; J(t)=j), n \geqslant 0 ; j=\overline{0, K}$, denote the steadystate probabilities of the process $\{(L(t) ; J(t)) ; t \geqslant 0\}$. The state-transition diagram is illustrated in Figure 1.

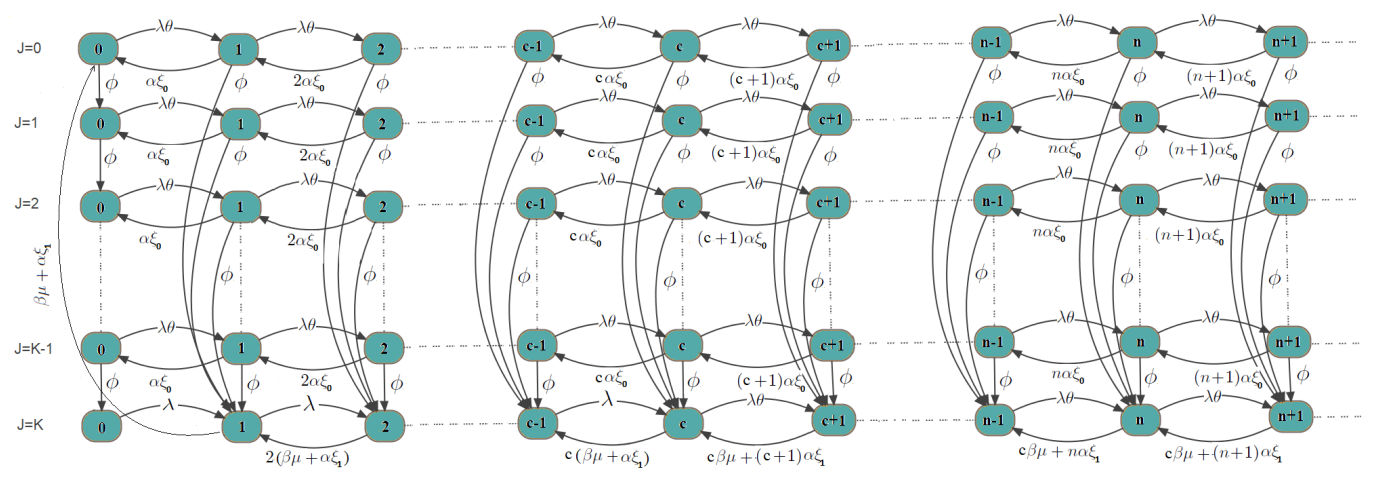

Figure 1. Transition plot

Using Chapman-Kolmogorov equations, we can formulate the balance equations for the suggested queueing model as:

$$
\begin{aligned}
& (\lambda+\phi) P_{0,0}=\alpha \xi_{0} P_{1,0}+\left(\beta \mu+\alpha \xi_{1}\right) P_{1, K}, \quad n=0, \\
& \left(\theta \lambda+\phi+\alpha \xi_{0}\right) P_{1,0}=\lambda P_{0,0}+2 \alpha \xi_{0} P_{2,0}, \quad n=1, \\
& \left(\theta \lambda+\phi+n \alpha \xi_{0}\right) P_{n, 0}=\theta \lambda P_{n-1,0}+(n+1) \alpha \xi_{0} P_{n+1,0}, \quad n \geqslant 2, \\
& (\lambda+\phi) P_{0, j}=\alpha \xi_{0} P_{1, j}+\phi P_{0, j-1}, \quad j=\overline{1, K-1}, \quad n=0, \\
& \left(\theta \lambda+\phi+\alpha \xi_{0}\right) P_{1, j}=\lambda P_{0, j}+2 \alpha \xi_{0} P_{2, j}, \quad j=\overline{1, K-1}, \quad n=1, \\
& \left(\theta \lambda+\phi+n \alpha \xi_{0}\right) P_{n, j}=\theta \lambda P_{n-1, j}+(n+1) \alpha \xi_{0} P_{n+1, j}, j=\overline{1, K-1}, n \geqslant 2 \text {, } \\
& \lambda P_{0, K}=\phi P_{0, K-1}, \quad n=0, \\
& \left(\lambda+\beta \mu+\alpha \xi_{1}\right) P_{1, K}=\lambda P_{0, K}+2\left(\beta \mu+\alpha \xi_{1}\right) P_{2, K}+\phi \sum_{j=0}^{K-1} P_{1, j}, \quad n=1, \\
& \left(\lambda+n\left(\beta \mu+\alpha \xi_{1}\right)\right) P_{n, K}= \\
& =\lambda P_{n-1, K}+(n+1)\left(\beta \mu+\alpha \xi_{1}\right) P_{n+1, K}+\phi \sum_{j=0}^{K-1} P_{n, j}, \quad 2 \leqslant n \leqslant c-1, \\
& \left(\theta \lambda+c \beta \mu+n \alpha \xi_{1}\right) P_{n, K}= \\
& =\lambda P_{n-1, K}+\left(c \beta \mu+(n+1) \alpha \xi_{1}\right) P_{n+1, K}+\phi \sum_{j=0}^{K-1} P_{n, j}, \quad n=c,
\end{aligned}
$$




$$
\begin{aligned}
& \left(\theta \lambda+c \beta \mu+n \alpha \xi_{1}\right) P_{n, K}= \\
& \quad=\theta \lambda P_{n-1, K}+\left(c \beta \mu+(n+1) \alpha \xi_{1}\right) P_{n+1, K}+\phi \sum_{j=0}^{K-1} P_{n, j}, \quad n>c .
\end{aligned}
$$

Consider the probability generating functions (PGFs) as:

$$
G_{j}(z)=\sum_{n=0}^{\infty} z^{n} P_{n, j}
$$

and define

$$
G_{j}^{\prime}(z)=\frac{d}{d z} G_{j}(z), \quad j=\overline{0, K}
$$

The normalizing condition is defined as

$$
\sum_{n=0}^{\infty} \sum_{j=0}^{K} P_{n, j}=1
$$

Multiplying Equation (3) by $z^{n}$, summing all possible values of $n$, and using Equations (1) and (2), we get

$$
\alpha \xi_{0}(1-z) G_{0}^{\prime}(z)-(\theta \lambda(1-z)+\phi) G_{0}(z)=-\left(\beta \mu+\alpha \xi_{1}\right) P_{1, K}+\bar{\theta} \lambda(1-z) P_{0,0} .
$$

In the same manner, from Equations (4)-(6) and (7)-(11) respectively, we obtain

$$
\begin{aligned}
& \alpha \xi_{0}(1-z) G_{j}^{\prime}(z)-[\theta \lambda(1-z)+\phi] G_{j}(z)= \\
& \quad=\bar{\theta} \lambda(1-z) P_{0, j}-\phi P_{0, j-1}, \quad j=\overline{1, K-1},
\end{aligned}
$$

and

$$
\begin{aligned}
& \alpha \xi_{1} z(1-z) G_{K}^{\prime}(z)-(1-z)(\theta \lambda z-c \beta \mu) G_{K}(z)= \\
& =c \beta \mu(1-z) P_{0, K}+z\left(\beta \mu+\alpha \xi_{1}\right) P_{1, K}-\phi z \sum_{j=0}^{K-1} G_{j}(z)+ \\
& \quad+\phi z \sum_{j=0}^{K-2} P_{0, j}+\lambda \bar{\theta} z(1-z) \Gamma_{1}(z)-\beta \mu(1-z) \Gamma_{2}(z),
\end{aligned}
$$

where

$$
\Gamma_{1}(z)=\sum_{n=0}^{c-1} z^{n} P_{n, K} \text { and } \Gamma_{2}(z)=\sum_{n=1}^{c-1}(n-c) z^{n} P_{n, K}
$$




\section{Solution of the differential equations}

For $z \neq 1$, Equation (12) can be written as follows:

$$
G_{0}^{\prime}(z)-\left[\frac{\theta \lambda}{\alpha \xi_{0}}+\frac{\phi}{\alpha \xi_{0}(1-z)}\right] G_{0}(z)=-\frac{\beta \mu+\alpha \xi_{1}}{\alpha \xi_{0}(1-z)} P_{1, K}+\frac{\bar{\theta} \lambda}{\alpha \xi_{0}} P_{0,0} .
$$

Multiply both sides of Equation $(15)$ by $e^{-\frac{\theta \lambda}{\alpha \xi_{0}} z}(1-z)^{\frac{\phi}{\alpha \xi_{0}}}$, we get

$$
\begin{aligned}
& \frac{d}{d z}\left(e^{-\frac{\theta \lambda}{\alpha \xi_{0}} z}(1-z)^{\frac{\phi}{\alpha \xi_{0}}} G_{0}(z)\right)= \\
& \quad=e^{-\frac{\theta \lambda}{\alpha \xi_{0}} z}(1-z)^{\frac{\phi}{\alpha \xi_{0}}}\left(\frac{\bar{\theta} \lambda}{\alpha \xi_{0}} P_{0,0}-\frac{\left(\beta \mu+\alpha \xi_{1}\right)}{\alpha \xi_{0}(1-z)} P_{1, K}\right) .
\end{aligned}
$$

Next, integrating the above equation from 0 to $z$, we obtain

$$
\begin{aligned}
G_{0}(z)=e^{\frac{\theta \lambda}{\alpha \xi_{0}} z}(1- & z)^{-\frac{\phi}{\alpha \xi_{0}} \times} \\
& \times\left\{G_{0}(0)+\frac{\bar{\theta} \lambda}{\alpha \xi_{0}} P_{0,0} C_{1}(z)-\frac{\beta \mu+\alpha \xi_{1}}{\alpha \xi_{0}} P_{1, K} C_{2}(z)\right\},
\end{aligned}
$$

with

$$
C_{1}(z)=\int_{0}^{z} e^{-\frac{\theta \lambda}{\alpha \xi_{0}} s}(1-s)^{\frac{\phi}{\alpha \xi_{0}}} d s \text { and } C_{2}(z)=\int_{0}^{z} e^{-\frac{\theta \lambda}{\alpha \xi_{0}} s}(1-s)^{\frac{\phi}{\alpha \xi_{0}}}-1 d s .
$$

Since $G_{0}(1)=\sum_{n=0}^{\infty} P_{n, 0}>0$ and $z=1$ is the root of denominator of the right hand side of Equation (16), we have that $z=1$ must be the root of the numerator of the right hand side of Equation (16). So, we obtain

$$
G_{0}(0)=\frac{\left(\beta \mu+\alpha \xi_{1}\right) P_{1, K}}{\alpha \xi_{0}} C_{2}(1)-\frac{\bar{\theta} \lambda P_{0,0}}{\alpha \xi_{0}} C_{1}(1)
$$

where

$$
C_{1}(1)=\int_{0}^{1} e^{-\frac{\theta \lambda}{\alpha \xi_{0}} s}(1-s)^{\frac{\phi}{\alpha \xi_{0}}} d s \text { and } C_{2}(1)=\int_{0}^{1} e^{-\frac{\theta \lambda}{\alpha \xi_{0}} s}(1-s)^{\frac{\phi}{\alpha \xi_{0}}}-1 d s .
$$

Noting $G_{0}(0)=P_{0,0}$. Then, Equation (17) implies

$$
P_{1, K}=\frac{\alpha \xi_{0}}{\left(\beta \mu+\alpha \xi_{1}\right) C_{2}(1)} B P_{0,0}=\varpi_{1} P_{0,0},
$$

with

$$
B=1+\frac{\lambda}{\alpha \xi_{0}} \bar{\theta} C_{1}(1) \text { and } \varpi_{1}=\frac{\alpha \xi_{0}}{\left(\beta \mu+\alpha \xi_{1}\right) C_{2}(1)} B
$$


Substituting Equation (18) into Equation (16), we obtain

$$
G_{0}(z)=e^{\frac{\theta \lambda}{\alpha \xi_{0}}} z(1-z)^{-\frac{\phi}{\alpha \xi_{0}}}\left\{1+\frac{\bar{\theta} \lambda}{\alpha \xi_{0}} C_{1}(z)-\frac{B}{C_{2}(1)} C_{2}(z)\right\} P_{0,0} .
$$

Next, Equation (13) can be written as

$$
G_{j}^{\prime}(z)-\left[\frac{\theta \lambda}{\alpha \xi_{0}}+\frac{\phi}{\alpha \xi_{0}(1-z)}\right] G_{j}(z)=\frac{\bar{\theta} \lambda}{\alpha \xi_{0}} P_{0, j}-\frac{\phi}{\alpha \xi_{0}(1-z)} P_{0, j-1} .
$$

Similarly, as for Equation (15), we multiply both sides of Equation (20) by $e^{-\frac{\theta \lambda}{\alpha \xi_{0}} z}(1-z)^{\frac{\phi}{\alpha \xi_{0}}}$. Then, we find

$$
\begin{aligned}
G_{j}(z) & =e^{\frac{\theta \lambda}{\alpha \xi_{0}} z}(1-z)^{-\frac{\phi}{\alpha \xi_{0}} \times} \\
& \times\left\{G_{j}(0)+\frac{\lambda \bar{\theta}}{\alpha \xi_{0}} C_{1}(z) P_{0, j}-\frac{\phi}{\alpha \xi_{0}} C_{2}(z) P_{0, j-1}\right\}, \quad j=\overline{1, K-1} .
\end{aligned}
$$

Since $G_{j}(1)=\sum_{n=0}^{\infty} P_{n, j}>0\left(G_{j}(1)=P_{\bullet}, j\right.$ represents the probability that the servers are taking the $(j+1)^{\text {th }}$ vacation) and $z=1$ is the root of denominator of the right hand side of Equation (21), we have that $z=1$ must be the root of the numerator of the right hand side of Equation (21). So, we obtain

$$
G_{j}(0)=P_{0, j}=A P_{0, j-1}, \quad j=\overline{1, K-1},
$$

where $A=\frac{\phi C_{2}(1)}{\alpha \xi_{0} B}$. Using Equation (22) repeatedly, we get

$$
P_{0, j}=A^{j} P_{0,0}, \quad j=\overline{1, K-1} .
$$

Now, by substituting Equation (23) into Equation (21), we find

$$
\begin{aligned}
G_{j}(z)=e^{\frac{\theta \lambda}{\alpha \xi_{0}}}(1-z)^{-\frac{\phi}{\alpha \xi_{0}}} A^{j} \times \\
\quad \times\left\{1+\frac{\lambda \bar{\theta}}{\alpha \xi_{0}} C_{1}(z)-\frac{B}{C_{2}(1)} C_{2}(z)\right\} P_{0,0}, \quad j=\overline{1, K-1} .
\end{aligned}
$$

To find $P_{0, K}$; the probability that the servers are idle during the busy period, we use Equations (7) and (23). Thus

$$
P_{0, K}=\varpi_{0} P_{0,0}
$$

where $\varpi_{0}=\frac{\phi}{\lambda} A^{K-1}$.

Remark 1. It is easy to see that $0<\phi C_{2}(1)<\alpha \xi_{0}$, and $\bar{\theta} \lambda C_{1}(1)>0$. Thus, $0<\phi C_{2}(1)<\alpha \xi_{0}+\bar{\theta} \lambda C_{1}(1)$. Consequently, we have $0<A<1$. 
Next, Equation (14) can be written as:

$$
\begin{aligned}
G_{K}^{\prime}(z)-\left(\frac{\theta \lambda}{\alpha \xi_{1}}-\right. & \left.\frac{c \beta \mu}{\alpha \xi_{1} z}\right) G_{K}(z)=\frac{\beta \mu+\alpha \xi_{1}}{\alpha \xi_{1}(1-z)} P_{1, K}+\frac{c \beta \mu}{\alpha \xi_{1} z} P_{0, K}+\frac{\lambda \bar{\theta}}{\alpha \xi_{1}} \Gamma_{1}(z)- \\
& -\frac{\beta \mu}{\alpha \xi_{1} z} \Gamma_{2}(z)+\frac{\phi}{\alpha \xi_{1}(1-z)}\left(\sum_{j=0}^{K-2} P_{0, j}-\sum_{j=0}^{K-1} G_{j}(z)\right)
\end{aligned}
$$

In the same way, by multiplying Equation $(13)$ by $\Upsilon(z)=e^{-\frac{\theta \lambda}{\alpha \xi_{1}} z} z^{\frac{c \beta \mu}{\alpha \xi_{1}}}$, we get

$$
\begin{array}{r}
\frac{d}{d z}\left(\Upsilon(z) G_{K}(z)\right)=\Upsilon(z)\left\{\frac{\beta \mu+\alpha \xi_{1}}{\alpha \xi_{1}(1-z)} P_{1, K}+\frac{c \beta \mu}{\alpha \xi_{1} z} P_{0, K}+\frac{\lambda \bar{\theta}}{\alpha \xi_{1}} \Gamma_{1}(z)-\right. \\
\left.-\frac{\beta \mu}{\alpha \xi_{1} z} \Gamma_{2}(z)+\frac{\phi}{\alpha \xi_{1}(1-z)}\left(\sum_{j=0}^{K-2} P_{0, j}-\sum_{j=0}^{K-1} G_{j}(z)\right)\right\} .
\end{array}
$$

Then, integrating from 0 to $z$ and using Equations (18) and (23)-(25), we obtain

$$
\begin{gathered}
G_{K}(z)=e^{\frac{\theta \lambda}{\alpha \xi_{1}} z} z^{-\frac{c \beta \mu}{\alpha \xi_{1}}}\left\{\left(\left(\beta \mu+\alpha \xi_{1}\right) \varpi_{1}+\phi\left(\frac{1-A^{K-1}}{1-A}\right)\right) H_{1}(z)+\right. \\
+\frac{c \beta \mu \phi}{\lambda} A^{K-1} H_{2}(z)-\phi\left(\frac{1-A^{K}}{1-A}\right) H_{3}(z)+\frac{1}{\alpha \xi_{1}} \times \\
\left.\times\left(\lambda \bar{\theta} \int_{0}^{z} s^{\frac{c \beta \mu}{\alpha \xi_{1}}} e^{-\frac{\theta \lambda}{\alpha \xi_{1}} s} \Gamma_{1}(s) d s-\beta \mu \int_{0}^{z} s^{\frac{c \beta \mu}{\alpha \xi_{1}}-1} e^{-\frac{\theta \lambda}{\alpha \xi_{1}} s} \Gamma_{2}(s) d s\right)\right\} P_{0,0}
\end{gathered}
$$

where

$$
\begin{gathered}
H_{1}(z)=\frac{1}{\alpha \xi_{1}} \int_{0}^{z} s^{\frac{c \beta \mu}{\alpha \xi_{1}}} e^{-\frac{\theta \lambda}{\alpha \xi_{1}} s}(1-s)^{-1} d s, \\
H_{2}(z)=\frac{1}{\alpha \xi_{1}} \int_{0}^{z} s^{\frac{c \beta \mu}{\alpha \xi_{1}}-1} e^{-\frac{\theta \lambda}{\alpha \xi_{1}} s} d s \\
H_{3}(z)=\frac{1}{\alpha \xi_{1}} \int_{0}^{z} s^{\frac{c \beta \mu}{\alpha \xi_{1}}} e^{-\frac{\theta \lambda}{\alpha \xi_{1}} s} \Psi(s)(1-s)^{-1} d s \\
\Psi(s)=e^{\frac{\theta \lambda}{\alpha \xi_{0}}} s(1-s)^{-\frac{\phi}{\alpha \xi_{0}}}\left\{1+\frac{\lambda \bar{\theta}}{\alpha \xi_{0}} C_{1}(s)-\frac{B}{C_{2}(1)} C_{2}(s)\right\} .
\end{gathered}
$$




\section{Evaluation of probabilities $P_{\bullet, K}, P_{\bullet, j}$ and $P_{0,0}$}

From Equations (18) and (25), we have $P_{1, K}=\varpi_{1} P_{0,0}$ and $P_{0, K}=\varpi_{0} P_{0,0}$. Making use of Equations (4)-(6), we recursively get

$$
\sum_{j=0}^{K-1} P_{n, j}=\delta_{n} P_{0,0},
$$

where

$$
\delta_{n}=\frac{1}{n \alpha \xi_{0}}\left\{\left[\theta \lambda+\phi+(n-1) \alpha \xi_{0}\right] \delta_{n-1}-\theta \lambda \delta_{n-2}\right\} .
$$

Similarly, from Equations (8), (9), we recursively obtain $P_{n, K}=\varpi_{n} P_{0,0}$, where

$$
\varpi_{n}=\frac{1}{n\left(\beta \mu+\alpha \xi_{1}\right)}\left\{\left[\lambda+(n-1)\left(\beta \mu+\alpha \xi_{1}\right)\right] \varpi_{n-1}-\lambda \varpi_{n-2}-\phi \delta_{n-1}\right\} .
$$

Thus, Equation (28) can be written as

$$
\begin{aligned}
& G_{K}(z)= \\
& \begin{aligned}
=e^{\frac{\theta \lambda}{\alpha \xi_{1}} z} z^{-\frac{c \beta \mu}{\alpha \xi_{1}}}\{[ & {\left[\frac{\alpha \xi_{0} B}{C_{2}(1)}+\phi\left(\frac{1-A^{K-1}}{1-A}\right)\right] H_{1}(z)+\frac{c \beta \mu \phi}{\lambda} A^{K-1} H_{2}(z)-} \\
& \left.-\phi\left(\frac{1-A^{K}}{1-A}\right) H_{3}(z)+\lambda \bar{\theta} H_{4}(z)-\beta \mu H_{5}(z)\right\} P_{0,0},
\end{aligned}
\end{aligned}
$$

with

$$
\begin{gathered}
H_{4}(z)=\frac{1}{\alpha \xi_{1}} \int_{0}^{z} s^{\frac{c \beta \mu}{\alpha \xi_{1}}} e^{-\frac{\theta \lambda}{\alpha \xi_{1}} s} \Theta_{1}(s) d s, \quad H_{5}(z)=\frac{1}{\alpha \xi_{1}} \int_{0}^{z} s^{\frac{c \beta \mu}{\alpha \xi_{1}}-1} e^{-\frac{\theta \lambda}{\alpha \xi_{1}} s} \Theta_{2}(s) d s \\
\Theta_{1}(z)=\sum_{n=0}^{c-1} z^{n} \varpi_{n}, \quad \text { and } \quad \Theta_{2}(z)=\sum_{n=1}^{c-1}(n-c) z^{n} \varpi_{n} .
\end{gathered}
$$

Thus, for $z=1$ (noting that $G_{K}(1)=P_{\bullet}, K$ represents the probability that the servers are busy or idle), we get

$$
G_{K}(1)=P_{\bullet, K}=\Phi(1) P_{0,0},
$$

where

$$
\begin{aligned}
\Phi(1) & =e^{\frac{\theta \lambda}{\alpha \xi_{1}}}\left\{\left(\left(\beta \mu+\alpha \xi_{1}\right) \varpi_{1}+\phi\left(\frac{1-A^{K-1}}{1-A}\right)\right) H_{1}(1)++\right. \\
& \left.+\frac{c \beta \mu \phi}{\lambda} A^{K-1} H_{2}(1)-\phi\left(\frac{1-A^{K}}{1-A}\right) H_{3}(1)+\lambda \bar{\theta} H_{4}(1)-\beta \mu H_{5}(1)\right\},
\end{aligned}
$$


with

$$
\begin{gathered}
H_{1}(1)=\frac{1}{\alpha \xi_{1}} \int_{0}^{1} s^{\frac{c \beta \mu}{\alpha \xi_{1}}} e^{-\frac{\theta \lambda}{\alpha \xi_{1}} s}(1-s)^{-1} d s, \\
H_{2}(1)=\frac{1}{\alpha \xi_{1}} \int_{0}^{1} s^{\frac{c \beta \mu}{\alpha \xi_{1}}-1} e^{-\frac{\theta \lambda}{\alpha \xi_{1}} s} d s, \\
H_{3}(1)=\frac{1}{\alpha \xi_{1}} \int_{0}^{1} s^{\frac{c \beta \mu}{\alpha \xi_{1}}} e^{-\frac{\theta \lambda}{\alpha \xi_{1}} s} \Psi(s)(1-s)^{-1} d s, \\
H_{4}(1)=\frac{1}{\alpha \xi_{1}} \int_{0}^{1} s^{\frac{c \beta \mu}{\alpha \xi_{1}}} e^{-\frac{\theta \lambda}{\alpha \xi_{1}} s} \Theta_{1}(s) d s, \\
H_{5}(1)=\frac{1}{\alpha \xi_{1}} \int_{0}^{1} s^{\frac{c \beta \mu}{\alpha \xi_{1}}-1} e^{-\frac{\theta \lambda}{\alpha \xi_{1}} s} \Theta_{2}(s) d s .
\end{gathered}
$$

Now, from Equations (12) and (13), for $z=1$, we have

$$
P_{\bullet, j}=G_{j}(1)=A^{j-1} P_{0,0}, \quad j=\overline{0, K-1} .
$$

By the definition of $P_{\bullet, j}$, using the normalizing condition, we get

$$
\sum_{j=0}^{K} P_{\bullet, j}=1 .
$$

Finally, from Equations (30) and (31), we get

$$
P_{0,0}=\left(\frac{1-A^{K}}{A(1-A)}+\Phi(1)\right)^{-1}
$$

\section{Performance measures}

The prime aim of determining probabilities in previous section is to formulate different metrics in order to examine the performance of the concerned system.

\subsection{Mean system sizes}

Systematic observations of the system state is very important to enhance the performance and to improve the decision-making.

Let $L_{j}$ be the system size when the servers are in the state $j(j=\overline{0, K})$. Thus, $\mathbb{E}\left(L_{j}\right)$ is the mean system size when the servers are in the state $j$, 
defined by

$$
\mathbb{E}\left(L_{j}\right)=G_{j}^{\prime}(1)=\sum_{n=1}^{\infty} n P_{n, j}, \quad j=\overline{0, K},
$$

that is, for $j=\overline{0, K-1}, \mathbb{E}\left(L_{j}\right)$ represents the mean system size when the servers are taking the $(j+1)^{\text {th }}$ vacation, and $\mathbb{E}\left(L_{K}\right)$ represents the mean system size when the servers are busy. We first derive $\mathbb{E}\left(L_{j}\right)$ for $j=\overline{0, K-1}$.

From Equation (15), using the Hospital rule, we get

$$
\begin{gathered}
\mathbb{E}\left(L_{0}\right)=G_{0}^{\prime}(1)= \\
=\lim _{z \rightarrow 1} \frac{-\theta \lambda G_{0}(z)+[\theta \lambda(1-z)+\phi] G_{0}^{\prime}(z)-\lambda \bar{\theta} P_{0,0}}{-\alpha \xi_{0}}= \\
=\frac{\theta \lambda G_{0}(1)-\phi G_{0}^{\prime}(1)+\bar{\theta} \lambda P_{0,0}}{\alpha \xi_{0}} .
\end{gathered}
$$

Thus, we get

$$
G_{0}^{\prime}(1)=\frac{\theta \lambda G_{0}(1)+\lambda \bar{\theta} P_{0,0}}{\alpha \xi_{0}+\phi}
$$

Similarly, from Equation (13), we find

$$
\left(\alpha \xi_{0}+\phi\right) G_{j}^{\prime}(1)=\theta \lambda G_{j}(1)+\lambda \bar{\theta} P_{0, j}, \quad j=\overline{1, K-1} .
$$

Then, from Equations (33) and (34), we have

$$
\mathbb{E}\left(L_{j}\right)=G_{j}^{\prime}(1)=\frac{\lambda\left[\theta G_{j}(1)+\bar{\theta} P_{0, j}\right]}{\alpha \xi_{0}+\phi}, \quad j=\overline{0, K-1} .
$$

By substituting Equation (31) and (35), we get

$$
\mathbb{E}\left(L_{j}\right)=\frac{\lambda}{\alpha \xi_{0}+\phi}\left[\frac{\theta+\bar{\theta} A}{A}\right] A^{j} P_{0,0}, \quad j=\overline{0, K-1} .
$$

Thus, the mean system size when the servers are on vacation is obtained as

$$
\begin{aligned}
\mathbb{E}\left(L_{V}\right)= & \sum_{j=0}^{K-1} \mathbb{E}\left(L_{j}\right)=\mathbb{E}\left(L_{0}\right)+\sum_{j=1}^{K-1} \mathbb{E}\left(L_{j}\right)= \\
=\frac{\lambda\left(\theta A^{-1}+\bar{\theta}\right)}{\left(\alpha \xi_{0}+\phi\right)} P_{0,0}+ & \frac{\lambda}{\left(\alpha \xi_{0}+\phi\right)}\left[\frac{\theta+\bar{\theta} A}{A}\right] \sum_{j=1}^{K-1} A^{j} P_{0,0}= \\
& =\left(\frac{\lambda(\theta+\bar{\theta} A)}{\alpha \xi_{0}+\phi}\right)\left\{\frac{2-\left(A+A^{K-1}\right)}{A(1-A)}\right\} P_{0,0} .
\end{aligned}
$$


Next, from Equation (26) and by using the Hospital rule, we get

$$
\begin{aligned}
& \mathbb{E}\left(L_{K}\right)=\lim _{z \rightarrow 1} G_{K}^{\prime}(z)= \\
& \begin{aligned}
\alpha \xi_{1}\left\{(\theta \lambda-c \beta \mu) \Phi(1)+c \beta \mu \frac{\phi}{\lambda} A^{K-1}\right. & \left.+\frac{\lambda \phi(\theta+\bar{\theta} A)}{\alpha \xi_{0}+\phi}\left(\frac{1-A^{K}}{A(1-A)}\right)\right\} P_{0,0}+ \\
& +\frac{1}{\alpha \xi_{1}}\left\{\bar{\theta} \lambda \Theta_{1}(1)-\beta \mu \Theta_{2}(1)\right\} P_{0,0},
\end{aligned}
\end{aligned}
$$

where $\Theta_{1}(1)=\sum_{n=0}^{c-1} \varpi_{n}$ and $\Theta_{2}(1)=\sum_{n=1}^{c-1}(n-c) \varpi_{n}$.

\subsection{Queueing model indices}

The expressions for the mean queue length, the mean number of customers served and the average rates of impatient customers are established as follows:

- The mean size of the queue is calculated as

$$
\begin{aligned}
\mathbb{E}\left(L_{q}\right)=\sum_{j=0}^{K-1} \sum_{n=1}^{\infty} n P_{n, j}+\sum_{n=c}^{\infty}(n-c) P_{n, K}= \\
=\mathbb{E}(L)-c+\left\{c\left[\frac{1-A^{K}}{A(1-A)}+\frac{\phi}{\lambda} A^{K-1}\right]-\Theta_{2}(1)\right\} P_{0,0} .
\end{aligned}
$$

- The mean number of customers served per unit of time is given as

$$
\begin{aligned}
E_{\mathrm{cs}}=\beta \mu \sum_{n=1}^{c-1} n P_{n, K} & +c \beta \mu \sum_{n=c}^{\infty} P_{n, K}= \\
= & \beta \mu\left\{c+\left[\Theta_{2}(1)-c\left(\frac{\phi}{\lambda} A^{K-1}+\frac{1-A^{K}}{A(1-A)}\right)\right] P_{0,0}\right\} .
\end{aligned}
$$

- The average rate of balking when the servers are in the state $j=\overline{0, K}$ is calculated as

$$
\begin{aligned}
B_{\mathrm{r}}=\bar{\theta} \lambda\left(\sum_{j=0}^{K-1} \sum_{n=1}^{\infty} P_{n, j}\right. & \left.+\sum_{n=c}^{\infty} P_{n, K}\right)= \\
& =\bar{\theta} \lambda\left\{1-\left[\frac{2-A-A^{K-1}+(1-A) \Theta_{1}(1)}{(1-A)}\right] P_{0,0}\right\} .
\end{aligned}
$$

- The average rate of abandonment of a customer due to reneging is as follows

$$
R_{\mathrm{ren}}=\sum_{j=0}^{K-1} \sum_{n=1}^{\infty} n \alpha \xi_{0} P_{n, j}+\sum_{n=1}^{\infty} n \alpha \xi_{1} P_{n, K}=\alpha \xi_{0} \mathbb{E}\left(L_{V}\right)+\alpha \xi_{1} \mathbb{E}\left(L_{K}\right) .
$$




\section{Conclusion}

In this paper, we studied an $M / M / c$ feedback queue under synchronous $K$-variant vacations, balking, server's states-dependent reneging and retention of reneged customers. We developed the Chapman-Kolmogorov equations for the steady-state probabilities and solved the differential equations by using the probability generating function method. Based on these results, we obtained the probability generating function of the number of customers in the system when the system is on vacation period (resp. on busy period). In addition, we derived explicit expressions of some useful performance measures for the system. Furthermore, we presented closed-form expressions of some important other queueing indices such as the probabilities when the servers are in different states, the proportion of customers served per unit of time, and the average rates of balking and reneging.

It would be interesting to investigate a similar model with two-phase services and multiple vacation policy, server breakdown and repair, and customers' impatience. Further, one can evaluate the optimality of service and repair rates to minimize the waiting time of the customers in the system.

\section{Acknowledgments}

The authors thank the anonymous referees for giving great interest to this article.

\section{References}

[1] M. Boualem, N. Djellab, and D. Aissani, "Stochastic Inequalities for an $M / G / 1$ retrial queues with vacations and constant retrial policy," Mathematical and Computer Modelling, vol. 50, no. 1-2, pp. 207-212, 2009. DOI: $10.1016 / \mathrm{j} . \mathrm{mcm} .2009 .03 .009$.

[2] R. Arumuganathan and K. S. Ramaswami, "Analysis of a bulk queue with fast and slow service rates and multiple vacations," Asia-Pacific Journal of Operational Research, vol. 22, no. 2, pp. 239-260, 2005. DOI: 10.1142/S0217595905000534.

[3] Y. Levy and U. Yechiali, "An M/M/s Queue With Servers' Vacations," INFOR: Information Systems and Operational Research, vol. 14, no. 2, pp. 153-163, 1976. DOI: 10.1080/03155986.1976.11731635.

[4] B. T. Doshi, "Queueing systems with vacations-a survey," Queueing Systems, vol. 1, no. 1, pp. 29-66, 1986. DOI: 10.1007/BF01149327.

[5] S. M. Gupta, "Machine interference problem with warm spares, server vacations and exhaustive service," Performance Evaluation, vol. 29, no. 3, pp. 195-211, 1997. DOI: 10.1016/S0166-5316(96)00046-6.

[6] N. Tian and Z. G. Zhang, Vacation queueing models: Theory and applications. New York, USA: Springer, 2006.

[7] Z. G. Zhang and N. Tian, "Analysis on queueing systems with synchronous vacations of partial servers," Performance Evaluation, vol. 52, no. 4, pp. 269-282, 2003. DOI: 10.1016/S0166-5316(02)00192-X. 
[8] Z. G. Zhang and N. Tian, "Analysis of Queueing Systems with Synchronous Single Vacation for Some Servers," Queueing Systems, vol. 45, pp. 161-175, 2003. DOI: 10.1023/A:1026097723093.

[9] A. A. Bouchentouf, M. Cherfaoui, and M. Boualem, "Performance and economic analysis of a single server feedback queueing model with vacation and impatient customers," OPSEARCH, vol. 56, pp. 300-323, 2019. DOI: $10.1007 / \mathrm{s} 12597-019-00357-4$.

[10] A. A. Bouchentouf, M. Cherfaoui, and M. Boualem, "Analysis and performance evaluation of Markovian feedback multi-server queueing model with vacation and impatience," American Journal of Mathematical and Management Sciences, vol. 40, no. 3, pp. 375-391, 2020. DOI: 10. 1080/01966324.2020.1842271.

[11] A. A. Bouchentouf and A. Guendouzi, "Sensitivity analysis of feedback multiple vacation queueing system with differentiated vacations, vacation interruptions and impatient customers," International journal of applied mathematics \&6 statistics, vol. 57, no. 6, pp. 104-121, 2018.

[12] A. A. Bouchentouf and A. Guendouzi, "The $M^{X} / M / c$ Bernoulli feedback queue with variant multiple working vacations and impatient customers: performance and economic analysis," Arabian Journal of Mathematics, vol. 9, pp. 309-327, 2020. DOI: 10.1007/s40065-0190260-x.

[13] M. Boualem, M. Cherfaoui, N. Djellab, and D. Aissani, "Analyse des performances du système $M / G / 1$ avec rappels et Bernoulli feedback," French, Journal Européen des Systèmes Automatisés, vol. 47, no. 1-3, pp. 181-193, 2013. DOI: 10.3166/jesa.47.181-193.

[14] A. Z. Melikov, S. H. Aliyeva, and M. O. Shahmaliyev, "Methods for computing a system with instantaneous feedback and variable input stream intensity," Automation and Remote Control, vol. 81, no. 9, pp. 16471658, 2020. DOI: 10.1134/S0005117920090052.

[15] A. A. Bouchentouf, M. Cherfaoui, and M. Boualem, "Modeling and simulation of Bernoulli feedback queue with general customers' impatience under variant vacation policy," International Journal of Operational Research, vol. 1, 2020. DOI: 10.1504/IJOR.2020.10034866.

[16] S. Benjaafar, J.-P. Gayon, and S. Tepe, "Optimal control of a productioninventory system with customer impatience," Operations Research Letters, vol. 38, no. 4, pp. 267-272, 2010. DOI: 10.1016/j.orl.2010.03.008.

[17] N. Gans, G. Koole, and A. Mandelbaum, "Telephone call centers: tutorial, review, and research prospects," Manufacturing and Service Operations Management, vol. 5, no. 2, pp. 79-141, 2003. DOI: 10.1287/msom.5.2. 79.16071.

[18] F. Afroun, D. Aïssani, D. Hamadouche, and M. Boualem, "Q-matrix method for the analysis and performance evaluation of unreliable $M / M / 1 / N$ queueing model," Mathematical Methods in the Applied Sciences, vol. 41, no. 18, pp. 9152-9163, 2018. DOI: 10.1002/mma.5119. 
[19] M. Boualem, "Stochastic analysis of a single server unreliable queue with balking and general retrial time," Discrete and Continuous Models and Applied Computational Science, vol. 28, no. 4, pp. 319-326, 2020. DOI: $10.22363 / 2658-4670-2020-28-4-319-326$.

[20] A. A. Bouchentouf, M. Cherfaoui, M. Boualem, and L. Medjahri, "Variant vacation queueing system with Bernoulli feedback, balking and server's states-dependent reneging," Yugoslav Journal of Operations Research, vol. 31, no. 4, pp. 1-19, 2021. DOI: 10.2298/YJOR200418003B.

[21] M. Manoharan and J. K. Jose, "Markovian queueing system with random balking," OPSEARCH, vol. 48, no. 3, pp. 236-246, 2011. DOI: 10.1007/ s12597-011-0054-1.

[22] E. Altman and U. Yechiali, "Analysis of customer's impatience in queues with server vacations," Queueing Systems, vol. 52, no. 4, pp. 261-279, 2006. DOI: $10.1007 / \mathrm{s} 11134-006-6134-\mathrm{x}$.

[23] E. Altman and U. Yechiali, "Infinite-server queues with systems additional task and impatient customers," Probability in the Engineering and Informational Sciences, vol. 22, no. 4, pp. 477-493, 2008. DOI: 10.1017/S0269964808000296.

[24] A. Economou, A. Gómez-Corral, and S. Kanta, "Optimal balking strategies in single-server queues with general service and vacation times," Performance Evaluation, vol. 68, no. 10, pp. 967-982, 2011. DOI: 10. 1016/j peva. 2011.07.001.

[25] A. A. Bouchentouf and A. Guendouzi, "Cost optimization analysis for an $M^{X} / M / c$ vacation queueing system with waiting servers and impatient customers," SeMA Journal, vol. 76, pp. 309-341, 2019. DOI: $10.1007 / \mathrm{s} 40324-018-0180-2$.

[26] W. Sun, S. Li, and E. Cheng-Guo, "Equilibrium and optimal balking strategies of customers in Markovian queues with multiple vacations and N-policy," Applied Mathematical Modelling, vol. 40, no. 1, pp. 284301, 2016. DOI: 10.1016/j.apm.2015.04.045.

[27] D. Yue, W. Yue, Z. Saffer, and X. Chen, "Analysis of an $M / M / 1$ queueing system with impatient customers and a variant of multiple vacation policy," Journal of Industrial and Management Optimization, vol. 10, no. 1, pp. 89-112, 2014. DOI: 10.3934/jimo.2014.10.89.

[28] D. Yue, W. Yue, and G. Zhao, "Analysis of an $M / M / c$ queueing system with impatient customers and synchronous vacations," Journal of Applied Mathematics, vol. 2014, 2014. DOI: 10.1155/2014/893094.

[29] D. Yue, Y. Zhang, and W. Yue, "Optimal performance analysis of an $M / M / 1 / N$ queue system with balking, reneging and server vacation," International Journal of Pure and Applied Mathematics, vol. 28, no. 1, pp. 101-115, 2006.

[30] D. Yue, W. Yue, and G. Zhao, "Analysis of an $M / M / 1$ queue with vacations and impatience timers which depend on the server's states," Journal of Industrial and Management Optimization, vol. 12, no. 2, pp. 653-666, 2016. DOI: 10.3934/jimo.2016.12.653. 


\section{For citation:}

A. A. Bouchentouf, L. Medjahri, M. Boualem, A. Kumar, Mathematical analysis of a Markovian multi-server feedback queue with a variant of multiple vacations, balking and reneging, Discrete and Continuous Models and Applied Computational Science 30 (1) (2022) 21-38. DOI: 10.22363/2658-4670-202230-1-21-38.

\section{Information about the authors:}

Bouchentouf, Amina Angelika - Full Professor, Professor of Mathematics at Djillali Liabes University of Sidi Bel Abbes, Algeria. Permanent researcher at Mathematics Laboratory of Sidi Bel Abbes (e-mail: bouchentouf amina@yahoo.fr, ORCID: https://orcid.org/0000-0001-8972-4221, ResearcherID: AAI-9425-2021, Scopus Author ID: 56682879900)

Medjahri, Latifa - Faculty Member at Abou Bekr Belkaid University of Tlemcen, Algeria (e-mail: 1.medjahri@yahoo.fr)

Boualem, Mohamed - Full Professor, Professor of Applied Mathematics at the Department of Technology at the University of Bejaia, Algeria. Permanent Researcher at the Research Unit LaMOS (Modeling and Optimization of Systems) (e-mail: robertt15dz@yahoo.fr, phone: +21334813708, ORCID: https://orcid.org/0000-0001-9414-714X, ResearcherID: A-3176-2019, Scopus Author ID: 26633399700)

Kumar, Amit - Assistant Professor at the Department of Mathematics at the University of Chandigarh, Mohali (Punjab), Pin No-140413, India (e-mail: amitk251@gmail.com, ORCID: https://orcid.org/0000-0001-5347-1808) 
УДК 519.872:519.217

PACS 07.05.Tp, 02.60.Pn, 02.70.Bf

DOI: $10.22363 / 2658-4670-2022-30-1-21-38$

\title{
Математический анализ марковской многолинейной системы массового обслуживания с обратной связью, прогулками приборов и нетерпеливыми заявками
}

\author{
Амина Анжелика Бушентуф ${ }^{1}$, Латифа Меджахри루, \\ Мохамед Буалем ${ }^{3}$, Амит Кумар ${ }^{4}$ \\ ${ }^{1}$ Университет Джиллали Лябеса в Сиди-Бель-Аббес, 22000, Алжир \\ ${ }^{2}$ Университет Тлемсан, 13000, Алюсир \\ 3 Университет Беджайя, 06000, Алжир \\ ${ }^{4}$ Университет Чандигар, Мохали (Пенджаб), 140413, Индия
}

\begin{abstract}
Аннотация. В работе исследуется система массового обслуживания с нетерпеливыми заявками, бернуллиевской обратной связью и прогулками приборов. В момент перед поступлением заявки в систему клиент, анализируя занятость системы и состояние приборов, принимает решение о принятии заявки или её уходе из системы. Предполагается, что нетерпение клиента может возникнуть как в период занятости, так и в период отдыха (прогулки) приборов из-за имевшихся ранее случаев длительного ожидания начала обслуживания в системе, информация о которых предоставляется с помощью определённого механизма. Обратная связь состоит в том, что часть ранее обслуженных клиентов может вернуться в систему для повторного обслуживания. Исследуемая система может применяться для анализа передачи данных в телекоммуникационных системах. Для стационарного распределения вероятностей записаны и решены с помощью производящих функций уравнения Колмогорова-Чепмена. Кроме того, получены аналитические выражения для ряда ключевых характеристик системы, например таких, как вероятности занятости или прогулки прибора, среднее число обслуженных заявок в единицу времени, средние интенсивности отказов от поступления и отказов от ожидания начала обслуживания.
\end{abstract}

Ключевые слова: марковская многолинейная система массового обслуживания, производящая функция, нетерпеливые заявки, прогулка прибора, обратная связь 